\title{
Phase-Locked Transmission Electron Microscopy for Detecting Dynamic Responses of Heterogeneous Materials and Electrochemical Devices under an Alternating Electric Potential
}

\author{
Seiji Takeda ${ }^{1}$, Kentaro Soma ${ }^{1}$, Ryotaro Aso ${ }^{1}$, Naoto Kamiuchi ${ }^{1}$ and Hideto Yoshida ${ }^{1}$ \\ ${ }^{1}$ The Institute of Scientific and Industrial Research, Osaka University, 8-1 Mihogaoka, Ibaraki, Osaka \\ 567-0047, Japan
}

Targets for dynamic characterization range from materials to devices with complex tailored structures on the nanoscale; for instance, composites of ferroelectric, metallic, semiconducting, and/or ion conducting materials. For structurally complex specimens, conventional spectroscopic methodology for dynamic characterization can only provide us with spatially averaged responses. We developed a new apparatus for transmission electron microscopy (TEM) [1] to acquire images and spectra, including TEM images, electron holograms, and electron energy loss spectra, synchronized with the measurement of the dynamic response of a specimen, for instance electrochemical impedance spectroscopy (EIS) under an applied AC electric potential (voltage and frequency, denoted $V_{\mathrm{AC}}$ and $f$, respectively) (Fig. 1). From a $V_{\mathrm{AC}}$ of frequency $f$, a shutter pulse signal is generated to open and close a pre-specimen shutter in a base TEM apparatus [1]. A pulse is generated per $V_{\mathrm{AC}}$ cycle from the targeted phase $\phi$ to $\phi+\Delta \phi$ with phase width $\Delta \phi(\Delta \phi<2 \pi) . \Delta \phi$ corresponds to the temporal pulse width $\tau(\tau<1 / f)$ of an electron beam; i.e., $\Delta \phi=2 \pi f \tau$. TEM images and electron holograms could thus be acquired at the phase locked from $\phi$ to $\phi+\Delta \phi$ with phase width $\Delta \phi(\Delta \phi<2 \pi)$ of an applied AC electric potential. The apparatus will be useful to characterize the dynamic properties of a specimen under an applied potential when combined also with the other ordinary TEM-related experimental techniques.

The methodology is especially useful for heterogeneous specimens. A phase-locked (strobe) TEM or electron-phase image is represented by $I(\mathrm{x}, \mathrm{y})_{f, \phi, \Delta \phi}\left(I_{f, \phi, \Delta \phi}\right.$ for short). Here $x$ and $y$ are the coordinates in an imaging plane. For a homogeneous material, $I(\mathrm{x}, \mathrm{y})_{f, \phi, \Delta \phi}$ is equal to a constant value at any $\phi, \Delta \phi$, and $f$; i.e., $I_{\mathrm{f}, \phi, \Delta \phi}=$ constant. Suppose simply that a composite made of two kinds of materials with different dynamic property to applied AC electric potential is investigated by phase-locked (strobe) TEM and electron holography. $I(\mathrm{x}, \mathrm{y})_{f, \phi, \Delta \phi}$ may become heterogeneous at a certain frequency $f_{\mathrm{c}}$ under an applied AC potential. For instance, EIS may reveal the specific frequencies $f_{\mathrm{c}}$ that are associated with the mobility of light ions in an all solid state electrochemical system, or an all-solid ion battery. Near and at $f_{\mathrm{c}}$, the mobility of ions changes markedly in the associated areas partially in a specimen, while that in the other areas remains unchanged. Dynamics of ions may induce lattice distortion and change the electrostatic potential distribution differently in the materials. Synchronized with an applied AC voltage with $f$ around $f_{\mathrm{c}}$, the intensity difference of images at a phase $\phi$ and anti-phase of $\phi$ or $\phi+\pi$ likely appears depending on the dynamic property of the materials.

$$
\delta I(x, y)_{f, \phi+\pi, \phi}=I(x, y)_{f, \phi+\pi}-I(x, y)_{f, \phi .}
$$

This equation is useful to identify the specific areas in a composite specimen that are responsible for the dynamics of ions.

When a base TEM apparatus used to implement phase-locked TEM possesses the sufficient spatial resolution, phase-locked TEM and electron holography could provide dynamic structural and electric 
change for the same region in a specimen under an applied AC electric potential. In our preliminary experiments [1], phase-locked (strobe) images and/or spectra are obtained for model specimens of polycrystalline aluminum and an all-solid-state lithium ion model battery (LIB). In the phase-locked TEM conditions, $f$ ranges from $1 \mathrm{~Hz}$ to about $40 \mathrm{kHz}$ and $\Delta \phi$ from $2 \pi / 80$ to $\pi$. $V_{\mathrm{AC}}$ ranges from $2 \mathrm{mV}$ to $1 \mathrm{~V}$ depending on observation conditions. The quality of phase-locked strobe images can be improved markedly using a phase-locked strobe electron beam. Under specific conditions, the spatial resolution in images is better than $0.12 \mathrm{~nm}$, even though the spatial resolution generally depends on $V_{\mathrm{AC}}, f$, the base TEM, and the conductivity of the specimen. For the model battery specimens, it is shown that electrochemical impedance spectroscopy and cyclic voltammetry can be performed in a TEM apparatus, and could potentially be synchronized with phase-locked (strobe) imaging and spectroscopy. It was found that severe electron irradiation damage was detected during phase-locked (strobe) electron holography of the model LIB specimens.

Finally, it is pointed out that the hardware and software to generate a preferential pulse shutter signal is crucially useful to control and/or diminish electron irradiation damage in a fragile specimen by controlling the pre-specimen shutter with temporal resolution better than $1 \mathrm{~ms}$, especially combined with a fast CMOS camera, regardless of the existence of applied AC electric potential.

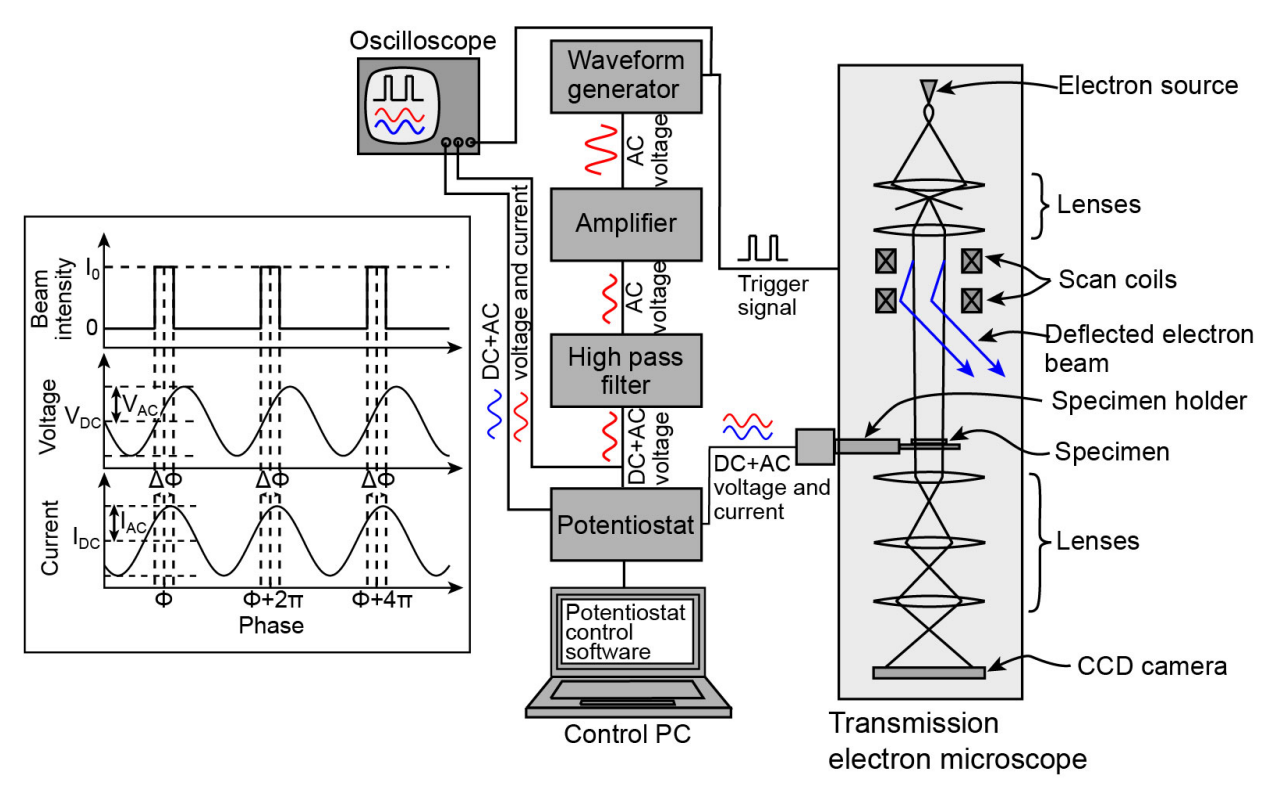

Figure 1. An apparatus for phase-locked TEM for detecting dynamic responses of materials and devices under an alternating electric potential [1]. Reprinted from Ultramicroscopy 181 (2017) 27-41, K. Soma et al., with permission from Elsevier.

References:

[1] K. Soma, S. Konings et al., Ultramicroscopy 181 (2017) 27-41.

[2] The authors acknowledge funding from SENTAN, JST and "Dynamic Alliance for Open Innovation Bridging Human, Environment and Materials" from the Ministry of Education, Culture, Sports, Science and Technology of Japan. 